Proceedings of SALT 26: 433-450, 2016

\title{
Hitting the nail on the head: Force vectors in verb semantics *
}

\author{
Anja Goldschmidt \\ UiL OTS, Utrecht University
}

\author{
Joost Zwarts \\ UiL OTS, Utrecht University
}

\begin{abstract}
We present an analysis of force verbs, like hit, as involving paths with force-dynamic properties, modeled through force vectors. This allows us to explain a number of observations about the lexical meaning and composition of these verbs. For instance, force adverbs such as hard specify the magnitude of the force vectors, while certain prepositions contribute information about their direction. The interpretation of resultative sentences with force verbs can also be explained. Resultative sentences like to hit the nail into the door are analyzed as complex events with caused results. This requires a force-dynamic computation with the force vectors involved in the first event, leading to the second event, and might explain otherwise mysterious incompatibilities of force modifiers and results that we observe for German.
\end{abstract}

Keywords: force dynamics, paths, force vectors, Neo-Davidsonian event semantics, hit, force verbs

\section{Introduction}

\subsection{Theoretical and empirical context}

There is a growing attention for the role of forces in the semantic analysis of verbs, in cognitive semantics (e.g. Talmy 2000; Croft 2012; Gärdenfors 2014); but also, more recently, in formal semantics (Van Lambalgen \& Hamm 2008; Copley \& Harley 2015; Roßdeutscher \& Pross 2015). One approach to force dynamics models forces as vectors located in space (e.g. Wolff 2007), providing a detailed analysis of the use of causal verbs like enable or prevent in motion situations, but without fitting those force vectors into a compositional semantics. Copley \& Harley (2015), on the other hand, offer a general compositional framework in which the event argument of any dynamic verb is reanalyzed as a force, defined more abstractly as a function

* We thank the SALT 26 organizers for making SALT 26 possible, NWO, Promoties in de Geesteswetenschappen for part of the funding that supported this research, and the audiences at the Düsseldorf SFB 991 General Colloquium, the Leiden Utrecht Semantics Happenings and the 17th Szklarska Poreba Workshop as well as our anonymous reviewers for helpful feedback on previous versions of this paper.

(C)2016 Goldschmidt \& Zwarts 
from situations to situations. Obviously, this creates a gap between the concrete, lexical-semantic notion of force vectors and their role in semantic composition.

We contribute towards bridging this gap by addressing a set of questions raised by force verbs in German, like schlagen (to hit), drücken (to push), ziehen (to pull). These verbs can be used to describe the exertion of a force on an object, without implying any change in the position or state of that object, cf. (1), although they can also have a resultative use, cf. (2). Other languages have similar verbs with similar properties, like the "impact verbs" in English (Jackendoff 1990; Levin 1993).

(1) Maria schlägt auf den Nagel.

Maria hits on the nail

'Maria hits the nail.'

(2) Maria schlägt den Nagel in die Wand.

Maria hits the nail in the wall

'Maria hits the nail into the wall.'

The resultative and non-resultative uses of force verbs raise a number of issues when analyzed in a standard event-semantic framework. We therefore propose to extend the event-based approach through explicit references to force vectors, drawing on insights of both cognitive and formal approaches to semantics and natural language meaning.

The paper is structured as follows. In the remainder of this section we will briefly outline the domain of force verbs. Section 2 then presents the problems that arise with a standard Neo-Davidsonian event-based analysis of force verbs and gives an outlook on how we plan to extend the analysis in order to solve these problems. Sections 3, 4 and 5 detail our analysis and show how the initial problems can be solved. Section 6 concludes.

\subsection{A domain of force verbs}

We use the following definition for 'force verb':

A force verb is any verb of which the root can occur in a sentence that describes a situation in which an object A (the agent) exerts a physical force (however light) ${ }^{1}$ on another object B (the patient) without necessarily implying a change in the properties of $\mathrm{B}$, yet while allowing for that change.

1 There is a close relationship between contact and force, as we use it: if one object exerts some force on another object, typically the two objects will be in contact (maybe via an intermediate entity). Correspondingly, when two objects are in contact, there is typically some amount of force exerted by one of the objects on the other. 
To illustrate: under the definition in (3), verbs like schlagen (to hit), drücken (to push), ziehen (to pull), pressen (to press), kneifen (to pinch), hämmern (to hammer) and treten (to kick) all count as force verbs. But verbs such as brechen (to break), werfen (to throw) and schleppen (to drag), on the one hand, do not count as force verbs because they all entail a change in the properties, including location, of the patient. Verbs such as stehen (to stand), on the other hand, do not count as force verbs because they don't allow for a change in the properties of the patient.

Despite the domain of force verbs being rather clear-cut, there is a lot of diversity of both lexical and compositional aspects. Concerning lexical meaning, force verbs can differ with respect to their aspectual properties. Compare e.g. drücken (to push), where the force is applied continuously, to schlagen (to hit), where the force is applied only punctually, i.e. non-continuously. Force verbs also differ with respect to directionality. The force can be directed towards the patient, as in e.g. drücken (to push), or away from the patient, as in e.g. ziehen (to pull). Other lexical distinctions include intensity (drücken ( $\sim$ to push) versus pressen $(\sim$ to press)), instrumental distinctions (treten (to kick - with a foot/leg) versus hämmern (to hammer) - with a hammer) and 'configurational' distinctions, i.e. whether the force is directed from two sides, as in e.g. kneifen (to pinch), or from one side, as in schlagen (to hit).

Force verbs also differ with respect to their combinatorial behavior, for example in their interactions with spatial prepositions. Compare e.g. *auf/ $\sqrt{ }$ an etwas ziehen (to pull $*$ on/ $\sqrt{ }$ on something) versus $\sqrt{ }$ auf/*an etwas drücken (to push $\sqrt{ }$ on/*on something). We also find alternations (cf. Levin 1993; Roßdeutscher \& Pross 2015): auf den Nagel schlagen (to hit on the nail) and den Nagel auf den Kopf schlagen (to hit the nail on the head) do not imply change while den Nagel in die Tür schlagen (to hit the nail into the door) does.

In the following, we will show how these distinctions can be worked into an extended Neo-Davidsonian analysis including not only events, but also paths and forces. This allows us to give a more accurate semantics of force verbs and to overcome a number of problems arising with the standard analysis of non-resultative uses versus resultative uses of force verbs, such as in (1) and (2) above.

\section{The (Neo-)Davidsonian starting point and beyond}

The sentences in (4a) and (4b) could get a standard analysis such as that in (5a) and (5b).

(4) a. Maria schlägt (hart) auf den Nagel.

Maria hits (hard) on the nail

'Maria hits the nail (hard).' 
b. Maria schlägt den Nagel (*hart) in die Wand.

Maria hits the nail (*hard) in the wall

'Maria hits the nail (*hard) into the wall.'
a. $\exists e[\operatorname{schlagen}(e) \wedge \operatorname{agent}(e)=\operatorname{maria} \wedge \operatorname{hart}(e) \wedge \operatorname{auf}(e, \operatorname{den}-n a g e l)]$
b. $\exists e[\operatorname{schlagen}(e) \wedge \operatorname{agent}(e)=\operatorname{maria} \wedge \operatorname{patient}(e$, den-nagel $) \wedge$ in $(e$, die- wand)]

However, this standard analysis leads to a number of problems.

Firstly, (4b) entails (4a). Every situation of Maria hitting the nail into the wall is a situation in which Maria hits the nail. But the reverse entailment doesn't hold: Maria could hit the nail without it moving. This is not accounted for by the logical forms in (5a) and (5b).

Secondly, den-nagel is the argument of the preposition auf in (5a), but the argument of the role patient in (5b), even though the nail plays a similar role in both, undergoing the hitting force.

Thirdly, based on (5a), we cannot account for the fact that schlagen (to hit) requires the preposition auf (on) in (4a), but not an (on), because nothing is specified about the relation between schlagen and auf (the same holds for ziehen (to pull) and an (on)).

Fourthly, the PPs in (4a) and (4b) make very different contributions to the meaning of the sentence. In (4a), auf den Nagel (on the nail) describes the movement of an instrument used by the agent (a hammer, presumably) onto the nail. In die Wand (into the wall) in (4b), on the other hand, describes the movement of the nail itself (resulting from the movement of the hammer onto the nail). These different contributions are not reflected in (5a) and (5b).

Fifthly, the standard analysis of the adverb hart (hard) in (4a) as hart(e) in (5a) does not specify what it is about an event of hitting that can be described as hard. Nor why this adverb is allowed in combination with schlagen (to hit), but not with e.g. verbs like essen (to eat).

Finally, as we already indicated with the asterisk in (4b), it seems that in German, when a force verb is used in a resultative construction, adverbs modifying the force, such as hart (hard) or leicht (lightly), are less accepted. This was confirmed by a questionnaire study with 158 German native speakers. The participants had to rate 20 test items (both resultative and non-resultative) on a 4-point Likert scale, ranging from 1 - clearly bad to 4 - clearly good. A general linear mixed-effects analysis shows that sentences that contain the force modifying adverbs hart or leicht but no resultative particle or preposition were judged significantly better than sentences with a resultative particle or preposition and a force modifying adverb (odds: 4.9, $\mathrm{p}$ $=.02$ for leicht; 8.1, $\mathrm{p}<.01$ for hart; percentages cf. Table 1). 


\begin{tabular}{|l|l|l|}
\hline- & without result & with result \\
\hline hart & $65 \%$ & $31 \%$ \\
\hline leicht & $80 \%$ & $55 \%$ \\
\hline
\end{tabular}

Table 1 Percentages of ratings 3 (maybe good) and 4 (clearly good) for all test sentences in the questionnaire

Our proposal is to extend the analysis in (5a) and (5b) to include the force patterns of events. More specifically, the internal properties of events are accessed through the paths they describe in real or conceptual space, as in e.g. Gärdenfors (2000). In line with Wolff (2007), we assume that such a path is constituted by a sequence of force vectors representing the force that the agent exerts on the patient at each point of time during the event. In the case of Maria hitting the nail (cf. (1)) there is in principle one non-zero force vector that corresponds with the actual, punctual contact between Maria's instrument (e.g. a hammer) and the nail. The length of that vector represents the magnitude of the force exerted through that contact. Before and after this point of contact, no forces are exerted by the hammer on the nail and therefore the corresponding vectors are zero. Zero-force vectors have a zero magnitude, but they are still located in space, i.e. they are points in space. This allows us to trace the movement of the hammer throughout the run time of the event (for more details see Section 3 below).

Following e.g. Talmy (2000) we further assume a rather general notion of path, which includes stationary positions as the limiting case. If a person is pushing against a car without moving it, for example, we would thus also speak of a path: the sequence of vectors that represent the forces that the agent exerts on (a particular part of) the car (the patient) at each point of time during the event. This means that even in a stationary situation where at every moment a force vector is generated in exactly the same place, we speak of a path.

Finally, we analyze resultative sentences as involving a representation with two events for caused results (cf. Parsons 1990; Pustejovsky 1991; and many following). In (4b) above we thus have one event associated to schlagen (to hit), and one event associated to in die Wand (into the wall).

At this point, these notions are all rather informal. In the next section, we will properly define our model-theoretic building blocks.

\section{Model-theoretic building blocks for a force-based approach}

Forces We assume a full set of located force vectors that have (i) an origin, (ii) a magnitude and (iii) a direction. This set includes zero force vectors, which have an 
origin, but zero magnitude and no direction. Each set of force vectors with the same spatial origin constitutes a vector space, with the corresponding algebraic properties, including addition and scalar multiplication.

Crucially, we access space exclusively through located force vectors. A zero force vector is equivalent with an "old-fashioned" point in space. A non-zero force vector $f$, on the other hand, can be used to represent a force with a magnitude $|f|$ working at point $\operatorname{ORIGIN}(f)=0 f$ (with 0 as a scalar).

Objects and space Objects are represented with a part-whole structure, e.g. a foot is part of the body.

Further, SPACE, BOUNDARY and INTERIOR are defined as follows, assuming convexity (cf. Zwarts \& Winter 2000):

(6) For every (material) object $x$, there is a set $\operatorname{SPACE}(x)$ of spatial points that represents the eigenspace of $x$ with a proper subset BOUNDARY $(x)$ of boundary points and INTERIOR $(x)$, the complement of the boundary with respect to the eigenspace (see Figure 3 for a visualization).

Paths A path is a continuous function from a time interval $\left[t_{0}, t_{1}\right]$ to the set of located force vectors (as described under Forces above). Roughly speaking, a path $p$ is a sequence of positions at which forces (possibly zero) are exerted:

(7) For every moment $t$ of the run time of an event $e: p(t)$ is the force vector $f$ representing the force exerted by the agent of $e$ on the patient of $e$ at time $t$.

As mentioned above, in accordance with Talmy (2000), a path $p$ may be constant, i.e. map every $t$ of its domain to the same vector $f$ (cf. 'pressing' in Figure $1 \mathrm{~b}$ and 'in' in Figure 1d).

We speak of a force path if a path includes non-zero force vectors in its range, i.e. there must be at least one moment during the event at which the agent is exerting a force on the patient (cf. 'hitting' and 'pressing' in Figure 1a and b, respectively). Otherwise we speak of a pure spatial path or path of motion (cf. 'into' and 'in' in Figure 1c and d, respectively).

Events in time and space We standardly assume a mereological structure of events to allow sums (+) of events. Events are mapped to time and space:

(8) For every event $e$, there is the interval $\operatorname{TimE}(e)$ that represents the running time of $e$.

(9) For any event $e, \operatorname{PATH}(e)$ is the path that corresponds to $e$, if defined. 


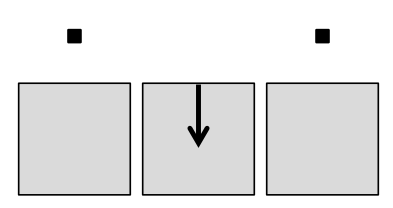

a. 'hitting'
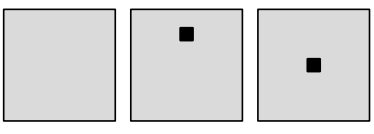

c. 'into'

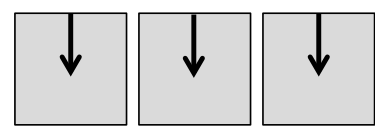

b. 'pressing'
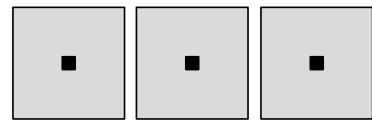

d. 'in'

Figure 1 Subsequent "snapshots" $\left(p\left(t_{0}\right), p\left(t_{i}\right), p\left(t_{1}\right)\right)$ from different paths.

Participants of events We operate with the following specifications for thematic roles or participants of events:

(10) If $\operatorname{PATH}(e)$ is a force path, then there is a participant $\operatorname{AGENT}(e)$ that is the exerter of the force and a participant $\operatorname{PATIENT}(e)$ that is the recipient of the force. Every non-zero force vector of the path must be located on the boundary of the eigenspace of $\operatorname{PATIENT}(e)$.

(11) If $\operatorname{PATh}(e)$ is a force path, then $\operatorname{InSTRUMENT}(e)$ is that part of $\operatorname{AgEnT}(e)$ that is in forceful contact with $\operatorname{PATIENT}(e)$.

(12) For any event $e$ with $\operatorname{PATH}(e)$, there is a participant $\operatorname{THEME}(e)$ that occupies subsequent positions of $\operatorname{PATH}(e)$ during the running time of $e$. This may be $\operatorname{INSTRUMENT}(e)$.

Causation In line with Wolff (2007), we characterize CAUSE $\left(e_{1}, e_{2}\right)$ in rough lines: ${ }^{2}$

(13) $\operatorname{CAUSE}\left(e_{1}, e_{2}\right)$ : if $\operatorname{PATH}\left(e_{1}\right)\left(t_{i}\right)+\operatorname{TENDENCy}\left(e_{1}\right)\left(t_{i}\right)$ is collinear with $\operatorname{PATH}\left(e_{2}\right)$ while TENDENCY $\left(e_{1}\right)\left(t_{i}\right)$ is not.

In words: $\operatorname{CAUSE}\left(e_{1}, e_{2}\right)$ holds if the patient's force tendency (TENDENCY $\left.\left(e_{1}\right)\left(t_{i}\right)\right)$ does not point in the direction of the path of the second event $\left(\operatorname{PATH}\left(e_{2}\right)\right)$, but the resultant of the agent's force $\left(\operatorname{PATH}\left(e_{1}\right)\left(t_{i}\right)\right)$ does. This captures Talmy's insight that

2 To keep things simple, we assume that it is possible to single out one moment $t_{i}$ of event $e_{1}$ that gives us the interacting forces of that event. We refrain from a definition of collinearity here. 


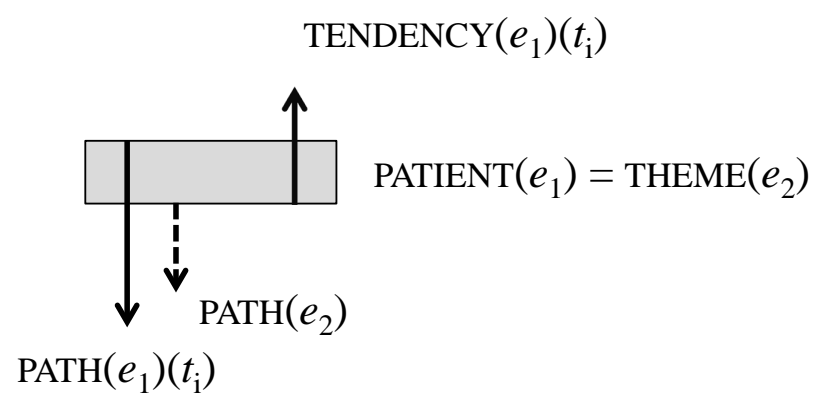

Figure 2 Force interaction in $\operatorname{CAUSE}\left(e_{1}, e_{2}\right)$ at moment $t_{i}$.

the agent (antagonist, in Talmy's terminology) in a causal interaction "overcomes" the tendencies of the patient (agonist, in Talmy's terminology).

Consider e.g. our previous example of a resultative use of a force verb, repeated here in (14). The force interaction in this case can be schematically modeled as in Figure 2.

(14) Maria schlägt den Nagel in die Wand.

Maria hits the nail into the wall.

The agent's force in this case is the force that Maria exerts on the nail at the moment of contact, represented with the non-zero vector $\left(\operatorname{PATH}\left(e_{1}\right)\left(t_{i}\right)\right)$ in Figure 2. The patient's force tendency (TENDENCY $\left.\left(e_{1}\right)\left(t_{i}\right)\right)$ subsumes all other forces working on the patient. These can be for example the density of the material of the wall, blocking the nail from moving into it, or gravity, if one wants to hit a nail into the ceiling. Crucially, the forces subsumed under TENDENCY $\left(e_{1}\right)\left(t_{i}\right)$ do not work in the same direction as the force in $\operatorname{PATH}\left(e_{1}\right)\left(t_{i}\right)$. Because the agent's force is higher than the patient's tendency, we get a resultant vector with the same direction as the path of motion $\left(\operatorname{PATH}\left(e_{2}\right)\right)$ of the patient (the nail), indicated in Figure 2 with the dashed arrow (not a vector, but a sequence of zero vectors, i.e. points). The force interaction in $e_{1}$ therefore counts as causing $e_{2}$. 
Hitting the nail on the head

\section{Meaning components and and lexical definitions}

The model-theoretic building blocks given in Section 3 can now be worked into the necessary meaning components and lexical definitions. In this section, we have chosen to illustrate only a subset of the domain of forces, namely only that subset that we will need to address the problems raised in Section 2.

Aspectual components As discussed in Section 1.2 above, force verbs can differ with respect to the aspectual components PUNCTUAL and CONTINUOUS, that we treat as properties of a path $p$ :

$$
\begin{aligned}
& \operatorname{PUNCTUAL}(p) \text { iff } \exists ! t[|p(t)|>0] \\
& \operatorname{CONTINUOUS}(p) \text { iff } \forall t[|p(t)|>0]
\end{aligned}
$$

In words: A path $p$ is punctual if and only if there is exactly one moment $t$ during the running time of the event at which the magnitude of the force vector is bigger than zero. A path $p$ is continuous if and only if for all moments $t$ during the running time of the event the magnitude of the force vector is bigger than zero.

Directional components Force verbs can also differ with respect to directionality (cf. Section 1.2). There are verbs like drücken (to push) and schlagen (to hit), where the force is directed towards the patient ("internally directed") and there are verbs like ziehen (to pull), where the force is directed away from the patient ("externally directed"). The definitions are as follows (illustrated in Figure 3):

$$
\begin{aligned}
& \operatorname{INTR}(p, x) \text { iff } \exists t . \exists \mathrm{s}[\operatorname{ORIGin}(p(t)) \in \operatorname{BOUNDARY}(x) \wedge \mathrm{s}>0 \wedge \operatorname{END}(\operatorname{sp}(t)) \in \\
& \operatorname{INTERIOR}(x)] \\
& \operatorname{EXTR}(p, x) \text { iff } \exists t . \forall \mathrm{s}[\operatorname{ORIGin}(p(t)) \in \operatorname{BOUNDARY}(x) \wedge \mathrm{s}>0 \wedge \operatorname{END}(\operatorname{sp}(t)) \notin \\
& \operatorname{SPACE}(x)]
\end{aligned}
$$

In words: a non-zero force vector $p(t)$ is internally directed with respect to an object $x$ if and only if there is a multiplied version (by a scalar s greater than zero) which has its endpoint in the interior of $x$. This makes both rightward directed vectors in Figure 3 internally directed vectors. For both vectors we can find some positive scalar multiple that brings the endpoint of the vector within the interior of that box-like object (as is already trivially the case with the shorter of the two vectors).

On the other hand, a non-zero force vector $p(t)$ is externally directed with respect to an object $x$ if and only if there is no multiplied version (by a scalar s greater than zero) which has its endpoint inside the space of $x$. This is true for the leftward directed vector in Figure 3. No matter which positive scalar multiple one applies, 


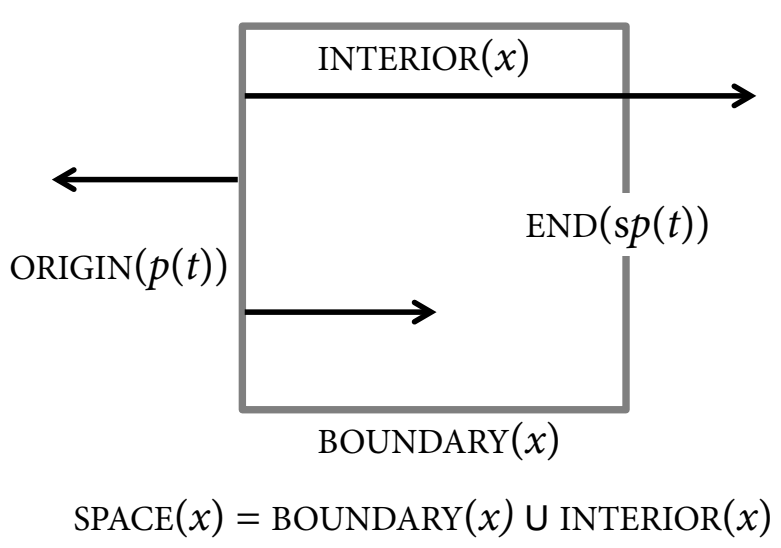

Figure $3 \operatorname{EXTR}(p, x)$ and $\operatorname{INTR}(p, x)$

the endpoint will never be part of the space of the box object (neither boundary nor interior).

Note that both types of force vectors have their origin at the boundary of the object $x$.

Force verbs With the meaning components properly defined, we are now able to put together the lexical definitions for force verbs. We focus here only on a set of three verbs, which were chosen because they illustrate prominent distinctions within the domain of force verbs: schlagen (to hit), drücken (to push) and ziehen (to pull).

$$
\begin{aligned}
& \operatorname{SCHLAGEN}=\lambda e . \exists p[p=\operatorname{PATH}(e) \wedge \operatorname{PUNCTUAL}(p) \wedge \operatorname{INTR}(p, \operatorname{PATIENT}(e))] \\
& \operatorname{DRÜCKEN}=\lambda e . \exists p[p=\operatorname{PATH}(e) \wedge \operatorname{CONTINUOUS}(p) \wedge \operatorname{INTR}(p, \operatorname{PATIENT}(e))] \\
& \operatorname{ZIEHEN}=\lambda e . \exists p[p=\operatorname{PATH}(e) \wedge \operatorname{CONTINUOUS}(p) \wedge \operatorname{EXTR}(p, \operatorname{PATIENT}(e))]
\end{aligned}
$$

Definitions 19-21 show how we build forces and paths into an event-based framework for verbs: the verb defines a set of events on the basis of properties of the corresponding force paths.

The aspectual distinctions are expressed through the "quantity" of non-zero force vectors, as defined in (15) and (16) above. For a punctual verb like schlagen, this means that there is only one moment of contact between agent and patient, during which the agent exerts a force on the patient. For continuous verbs like drücken and ziehen, on the other hand, agent and patient are in contact throughout the event, and the agent continuously exerts force on the patient.

The directional distinctions are expressed through the direction of the non-zero force vectors. In the case of schlagen and drücken, the force is directed towards the 
Hitting the nail on the head

patient. In the case of ziehen, on the other hand, the force is directed away from the patient.

Prepositions Prepositions are likewise defined in terms of forces and paths. We chose four prepositions to illustrate this: auf (on), gegen (against), an (on) and in (in/into). Their lexical definitions are the following:

$$
\begin{aligned}
& \operatorname{AUf}(x)=\lambda p[\operatorname{INTR}(p, x)] \\
& \operatorname{Gegen}(x)=\lambda p[\operatorname{INTR}(p, x)]^{3} \\
& \operatorname{AN}(x)=\lambda p[\operatorname{EXTR}(p, x)] \\
& \operatorname{IN}(x)=\lambda p\left[p\left(t_{1}\right) \in \operatorname{InTERioR}(x)\right]^{4}
\end{aligned}
$$

Note that according to the definitions in (22-25), prepositions are treated as denoting sets of paths, not sets of events. ${ }^{5}$ The directional components re-appear here. Auf and gegen are defined by internally directed force vectors, while an is defined by externally directed force vectors. In, on the other hand, has a condition attached to the last vector of its path, namely that this is a zero vector in the interior of the complement of $i$.

Force adverbs Finally, we can also give the lexical definitions of adverbs relating to force, such as hart (hard) and leicht (lightly). Like prepositions, these adverbs denote sets of paths.

$$
\begin{aligned}
& \text { HART }=\lambda p . \exists t\left[|p(t)|>\mathrm{M}_{c}\right] \\
& \text { LEICHT }=\lambda p . \forall t\left[|p(t)|<\mathrm{M}_{c}\right]
\end{aligned}
$$

The notation $\mathrm{M}_{c}$ in the definitions in $(26+27)$ stands for some average magnitude for comparison. For a force path to count as hart, there must be at least one vector whose magnitude is higher than some average for comparison. For a force path to count as leicht, on the other hand, the magnitude of all its vectors must be lower than some average for comparison.

\section{The compositional semantics}

\section{$5.1 \quad$ Type shifts}

With the lexical entries in Section 4 in place, we are almost ready to compositionally build up the denotations of the sentences in (4a) and (4b), cf. the non-resultative

3 For practical purposes, auf and gegen receive the same analysis here, despite their different uses.

4 This definition might not account for all occurrences of in. E.g. in das Kissen schlagen ("to hit into the pillow") does not require the force path to end in the interior of the pillow.

5 We will introduce a type shift to event denotations later on. 
and resultative uses of force verbs. However, there is a type mismatch between the verbs on the one hand, denoting sets of events, and the prepositions and adverbs on the other hand, which are defined as denoting sets of paths. $\mathrm{PP}+\mathrm{V}$ and Adverb+V combinations will now result in a type clash, and this needs to be solved first. In order to do this, we introduce two type-shift functions.

$$
\begin{aligned}
& \lambda P . \lambda E . \lambda e . \exists p[\operatorname{PATH}(e)=p \wedge P(p) \wedge E(e)] \\
& \lambda P . \lambda E . \lambda x \cdot \lambda e \cdot \exists e_{1} \cdot \exists e_{2} \cdot \exists p\left[e=e_{1}+e_{2} \wedge \operatorname{CAUSE}\left(e_{1}, e_{2}\right) \wedge E\left(e_{1}\right) \wedge\right. \text { PATIENT } \\
& \left.\left(e_{1}\right)=\operatorname{THEME}\left(e_{2}\right)=x \wedge \operatorname{PATH}\left(e_{2}\right)=p \wedge P(p)\right]
\end{aligned}
$$

The type shift in (28) takes a set of paths and maps it to a modifier of events, allowing PPs and adverbs to restrict sets of events to those events that are assigned to a particular path, as specified by the preposition or adverb.

The type shift in (29) is a complex predicate constructor. ${ }^{6}$ We need this type shift for sentences containing force verbs in the resultative construction, such as (4b). This type shift takes a PP denotation $P$ and a $\mathrm{V}$ denotation $E$ and maps those to a relation that holds between an object $x$ and an event $e$ if $x$ moves in $e$ along a $P$ path as the result of an $E$ force working on it.

Finally, we assume a function similar to that of Kratzer (1996) for introducing the external argument corresponding to the agent and existentially closing the event variable.

\subsection{Derivations}

The derivations of the sentences in (4a) and (4b), repeated below as (30) and (32), are as follows.

(30) Maria schlägt hart auf den Nagel

Maria hits hard on the nail

$$
\begin{aligned}
& \text { a. } \operatorname{schlagen}=\lambda e[\operatorname{SCHLAGEN}(e)] \\
& \text { b. auf }=\lambda x \cdot \lambda p[\operatorname{AUF}(p, x)] \\
& \text { c. auf den Nagel }=\lambda p[\operatorname{AUF}(p, \text { den-nagel })] \Rightarrow \lambda E \cdot \lambda e \cdot \exists p[\operatorname{PATH}(e)=p \wedge \\
& \quad \operatorname{AUF}(p, \text { den-nagel }) \wedge E(e)] \\
& \text { d. auf den Nagel schlagen }=\lambda e \cdot \exists p[\operatorname{PATH}(e)=p \wedge \operatorname{AUF}(p, \text { den-nagel }) \wedge \\
& \quad \operatorname{SCHLAGEN}(e)] \\
& \text { e. hart }=\lambda p[\operatorname{HART}(p)] \Rightarrow \lambda E \cdot \lambda e \cdot \exists p[\operatorname{PATH}(e)=p \wedge \operatorname{HART}(p) \wedge E(e)] \\
& \text { f. hart auf den } \operatorname{Nagel} \operatorname{schlagen}=\lambda e \cdot \exists p[\operatorname{PATH}(e)=p \wedge \operatorname{HaRT}(p) \wedge \operatorname{AUF}(p, \\
& \quad \text { den-nagel }) \wedge \operatorname{SCHLAGEN}(e)]
\end{aligned}
$$

6 Please note that a small-clause analysis could do the same work here. As far as this paper is concerned, a complex predicate approach and a small clause approach are both equally valid. 
Hitting the nail on the head

g. Maria schlägt hart auf den Nagel $=\exists e \cdot \exists p[\operatorname{PATH}(e)=p \wedge \operatorname{HART}(p) \wedge$ $\operatorname{AUF}(p$, den-nagel $) \wedge \operatorname{SCHLAGEN}(e) \wedge \operatorname{AGENT}(e)=$ MARIA $]$

Maria schlägt den Nagel in die Wand

Maria hits the nail into the wall

a. $\operatorname{schlagen}=\lambda e[\operatorname{SCHLAGEN}(e)]$

b. in $=\lambda x \cdot \lambda p[\operatorname{IN}(p, x)]$

c. in die Wand $=\lambda p[\operatorname{IN}(p$, die-wand $)] \Rightarrow \lambda E \cdot \lambda x \cdot \lambda e \cdot \exists e_{1} \cdot \exists e_{2} \cdot \exists p\left[e=e_{1}+\right.$ $e_{2} \wedge \operatorname{CAUSE}\left(e_{1}, e_{2}\right) \wedge E\left(e_{1}\right) \wedge \operatorname{PATIENT}\left(e_{1}\right)=\operatorname{THEME}\left(e_{2}\right)=x \wedge \operatorname{PATH}\left(e_{2}\right)$ $=p \wedge \mathrm{IN}(p$, die-wand $)]$

d. in die Wand schlagen $=\lambda x \cdot \lambda e \cdot \exists e_{1} \cdot \exists e_{2} \cdot \exists p\left[e=e_{1}+e_{2} \wedge \operatorname{CAUSE}\left(e_{1}, e_{2}\right) \wedge\right.$ $\operatorname{SChLAGEN}\left(e_{1}\right) \wedge \operatorname{PATIENT}\left(e_{1}\right)=\operatorname{THEME}\left(e_{2}\right)=x \wedge \operatorname{PATH}\left(e_{2}\right)=p \wedge \operatorname{IN}(p$, die-wand) ]

e. den Nagel in die Wand schlagen $=\lambda e \cdot \exists e_{1} \cdot \exists e_{2} \cdot \exists p\left[e=e_{1}+e_{2} \wedge \operatorname{CAUSE}\left(e_{1}\right.\right.$, $\left.e_{2}\right) \wedge \operatorname{SCHLAGEN}\left(e_{1}\right) \wedge \operatorname{PATIENT}\left(e_{1}\right)=\operatorname{THEME}\left(e_{2}\right)=$ den-nagel $\wedge \operatorname{PATH}\left(e_{2}\right)$ $=p \wedge \operatorname{IN}(p$, die-wand $)]$

f. Maria schlägt den Nagel in die Wand $=\exists e \cdot \exists e_{1} \cdot \exists e_{2} \cdot \exists p\left[e=e_{1}+e_{2} \wedge\right.$ $\operatorname{CAUSE}\left(e_{1}, e_{2}\right) \wedge \operatorname{SCHLAGEN}\left(e_{1}\right) \wedge \operatorname{PATIENT}\left(e_{1}\right)=\operatorname{THEME}\left(e_{2}\right)=$ den-nagel $\wedge \operatorname{PATH}\left(e_{2}\right)=p \wedge \operatorname{IN}(p$, die-wand $\left.) \wedge \operatorname{AGENT}\left(e_{1}\right)=\operatorname{MARIA}\right]$

The derivations in (31) and (33) show how the type shifts discussed in Section 5.1 above make the composition work. To derive the denotation of the non-resultative example in (30), the event modifier type shift in (28) is selected twice, shifting the PP auf den Nagel (on the nail) and the adverb hart (hard) from sets of paths to event modifiers. For the resultative use of force verbs in (32), the complex predicate constructor in (29) is selected. It shifts the PP in die Wand (into the wall) from a set of paths to a complex predicate constructor, which lays the relation between the "causing" event $e_{1}$ (to which schlagen applies) and the "caused" event $e_{2}$ (to which in die Wand applies).

\subsection{Answering the puzzles}

In Section 2 we laid out six problems that arise from the standard Neo-Davidsonian event-based treatment of non-resultative and resultative uses of force verbs. We will now demonstrate step by step how our extended Neo-Davidsonian analysis as laid out in Sections 3 to 5.2 allows us to solve all the issues.

Entailment There is an entailment from $(4 b) /(32)$ to $(4 a) /(30)$, but not vice versa, because in order to hit the nail into the wall, Maria must have hit it, yet she could 
have hit it without it moving into the wall. Previously, we were unable to account for this.

Now, if we take a close look at the lexical definitions in Section 4 and the derivations above, we can see that the entailment is due to the fact that SCHLAGEN specifies a path with internally directed force vectors with respect to the patient (cf. the definition for schlagen (to hit) in (19)), the same that AUF expresses (cf. the definition for auf (on) in (22)). Our analysis guarantees that schlagen always implies auf, roughly speaking.

This can be made explicit when we take the final result of the derivation in (33), drop a few conditions (via the logical rule of simplification), and then add the entailment from $\operatorname{SCHLAGEN}\left(e_{1}\right)$ to $\operatorname{INTR}\left(p, \operatorname{PATIENT}\left(e_{1}\right)\right)$, arriving at the denotation of (30) (minus hart (hard)).

$$
\begin{aligned}
& \exists e . \exists e_{1} \cdot \exists e_{2} \cdot \exists p\left[e=e_{1}+e_{2} \wedge \operatorname{CAUSE}\left(e_{1}, e_{2}\right) \wedge \operatorname{SCHLAGEN}\left(e_{1}\right) \wedge \operatorname{PATIENT}\left(e_{1}\right)\right. \\
& =\operatorname{THEME}\left(e_{2}\right)=\text { den-nagel } \wedge \operatorname{PATH}\left(e_{2}\right)=p \wedge \operatorname{IN}(p, \text { die-wand }) \wedge \operatorname{AGENT}\left(e_{1}\right) \\
& =\operatorname{MARIA}] \\
& \Rightarrow \exists e_{1}\left[\operatorname{SCHLAGEN}\left(e_{1}\right) \wedge \operatorname{PATIENT}\left(e_{1}\right)=\text { den-nagel } \wedge \operatorname{AGENT}\left(e_{1}\right)=\operatorname{MARIA}\right] \\
& \Rightarrow \exists e_{1} \cdot \exists p\left[\operatorname{PATH}\left(e_{1}\right)=p \wedge \operatorname{SCHLAGEN}\left(e_{1}\right) \wedge \operatorname{PATIENT}\left(e_{1}\right)=\mathbf{d e n}-\mathbf{n a g e l} \wedge\right. \\
& \left.\operatorname{AGENT}(e)=\operatorname{MARIA} \wedge \operatorname{INTR}\left(p, \operatorname{PATIENT}\left(e_{1}\right)\right)\right]
\end{aligned}
$$

The entailment does not hold in reverse, because the resultative sentence also contains a second event with different force vector properties that are not present in the event in the non-resultative sentence.

Patient The second puzzle concerns the role of the DP den Nagel (the nail). In $(4 a) /(30)$ this is the argument of the preposition auf (on). But in $(4 b) /(32)$, the same phrase is the patient of the verb. The close relation between these two notions is again explained through the underlying force vector and path properties of the verbs and prepositions involved.

The solution in this case lies in our definition of patient, cf. (10). We state that if there is a force path associated to an event $e$, then there is a participant $\operatorname{PATIENT}(e)$ that is the recipient of the force. And further: every non-zero force vector of the path $p$ of $e$ must be located on the boundary of the eigenspace of that PATIENT(e). Applying this to the phrase den Nagel, we can see that the conditions for "patienthood" are met in both cases. In both cases we are dealing with a force path $(\operatorname{PATH}(e)$ in (30) and $\operatorname{PATH}\left(e_{1}\right)$ in (32)), and in both cases, the nail is the recipient of that force, with the origin of the non-zero force vector located on the boundary of the nail.

The solution to this problem is thus to have definitions of thematic roles (patient) and prepositions (auf) based on the same theoretical foundation of forces. 
Hitting the nail on the head

Preposition selection The next puzzle concerns the selectional restrictions some verbs display with respect to prepositions. To recapitulate, a verb like schlagen (to hit) requires the preposition auf(on), whereas ziehen (to pull) requires the preposition an (on).

These selectional restrictions are explained through the need to match the direction of the force vectors of the path assigned to the event with those of the set of paths denoted by the PP to arrive at a non-empty set-intersection. The PP auf den Nagel (on the nail) is treated as a set of paths with internally directed force vectors applying to the nail (the patient), as specified in (22), cf. the definition for auf. The PP an dem Nagel (on the nail), on the other hand, is treated as a set of paths with externally directed force vectors, again applying to the patient, as defined for an in (24). These PPs thus have oppositely directed force vectors. Since the PP+V combinations (after the PPs are shifted to sets of events, retaining their paths' properties) are interpreted as intersections, auf den Nagel schlagen leads to a non-empty intersection because auf and schlagen require the same directional properties, i.e. internally directed force vectors. But \#an dem Nagel schlagen leads to an empty intersection, because the direction of the force vectors of an is the opposite of those of schlagen. The reverse pattern holds for an dem Nagel ziehen and \# auf dem Nagel ziehen.

Prepositional phrases Under the standard event-based analysis it is also unclear how to differentiate between the meaning contributions of the prepositional phrases auf den Nagel (on the nail) and in die Wand (into the wall), since both are treated as predicates over events. Again, this can be solved by appealing to the different force properties of their respective paths.

The contributions made by these PPs can be distinguished by the different types of paths assigned to them. Auf den Nagel, with the denotation $\lambda p$ [ INTR( $p$,dennagel) ], specifies a force path (as in Figure 1a+b). In die Wand, however, has the denotation $\lambda p\left[p\left(t_{1}\right) \in \operatorname{INTERIOR(die-wand)],~describing~motion~events~into~the~}\right.$ wall (cf. Figure 1c), without any specification of forces.

Force adverbs 1 There is also a problem with respect to so-called force adverbs. In the analysis in $(5 a), \operatorname{hart}(e)$, it is not clear what part of the event is specified as hard, nor why this adverb is not a good modifier for all verbs, cf. \# hart essen (to eat hard).

With the definitions of hart (hard) and leicht (lightly) in (26) and (27) respectively, the first part of this puzzle is now easy to solve. According to those definitions, what it means for an event to be hard is that the magnitude of some force vector in the path of the event is higher than a certain average. Similarly, an event is light if all force vectors constituting the path of the event have a magnitude below a 
certain average. It is thus properties of paths of events and their force vectors that the adverbs hart and leicht refer to.

This also leads us straight to the answer of the second part of this puzzle. Force verbs like schlagen (to hit) as defined in (3) have a path and corresponding force vectors assigned to them, which is what allows modification by adverbs such as hart and leicht that modify these vectors. Other verbs, such as e.g. the above mentioned essen, are not associated to force paths. There is thus nothing in the lexical semantics of these verbs to be modified by force-related adverbs.

Force adverbs 2 The final puzzle concerns the apparent "unmodifiability" of force verbs in resultative use through force-related adverbs. To recapture, the questionnaire results, summarized in Table 1, show that sentences with force verbs in the resultative construction and modified by a force-related adverb (cf. (4b), repeated as (35b) below) are judged significantly worse than sentences with force verbs in the nonresultative construction and modified by a force-related adverb (cf. (4a), repeated as (35a) below).

a. Maria schlägt (hart) auf den Nagel. Maria hits (hard) on the nail

'Maria hits the nail hard.'

b. Maria schlägt den Nagel (*hart) in die Wand.

Maria hits the nail (*hard) in the wall

'Maria hits the nail (*hard) into the wall.'

This can now be explained as follows: In (13) in Section 3 above, under the heading Causation, we defined CAUSE and explained the underlying force interaction of this concept. This interaction requires an operation of vector addition of the force (vector) exerted by the agent on the patient at the moment of contact between the two, $\operatorname{PATH}\left(e_{1}\right)\left(t_{i}\right)$, and the patient's tendencies, TENDENCY $\left(e_{1}\right)\left(t_{i}\right)$, resulting in a vector pointing in the direction of the path of motion $\operatorname{PATH}\left(e_{2}\right)$. We suggest that, once this computation has happened, the original force vectors of $\mathrm{PATH} e_{1}$ become unavailable for modification by any adverb that requires a force vector with a magnitude (bigger than zero). The only available force vectors are those of $\operatorname{PATH}\left(e_{2}\right)$, which is a path of motion and has therefore only zero force vectors without a magnitude. This path is thus not a suitable "attachment site" for force-related adverbs.

Please note that adverbs such as schief (diagonally), gerade (straight), schnell (quickly), langsam (slowly), vorsichtig (carefully) and hektisch (hastily) are all allowed as modifiers in the resultative sentences. The first pair (schief-gerade) modifies the form of the resulting path of motion. The second pair (schnell-langsam) modifies the complex event $\left(e=e_{1}+e_{2}\right)$, i.e. the time that it takes until the result is 
reached. The last pair (vorsichtig - hektisch) modifies the manner of the first event and is licensed by the agent (i.e. only an animate agent can do things in a hasty or careful manner).

\section{Summary and Conclusion}

This paper presented a partial analysis of German force verbs: verbs that can be used to describe the exertion of a force on an object, leaving open the possibility of but not requiring a change in the position or state of that object.

A standard event-based analysis of these verbs, that ignores the role of forces, presents a number of problems which can be solved as soon as we make explicit reference to forces, formalisable as vectors. In short: internal properties of events, such as their ability to be "hard" or to involve "impact", are accessed via paths (cf. Gärdenfors 2000; Talmy 2000) that describe the way the force exertion (by the agent on the patient) develops over the course of the event, namely as a sequence of positions at which a force could be exerted.

It is those vectors and their properties that help us to account not only for the distinctions we find in the domain of force verbs, such as differences in the aspectual, directional or compositional aspects. But also for issues such as the entailment of the non-resultative use of a force verb in its resultative use or the distinct meaning contributions of superficially similar prepositional phrases. The analysis thus allows for a compositional semantics of sentences/phrases with force verbs that does justice to relevant lexical factors and thereby bridges the gap between the lexical/conceptual side of meaning and the grammatical/compositional side.

Our analysis also opens up wider empirical and theoretical possibilities in the area of force-dynamics, with broader and deeper analyses of verbs, adjectives/adverbs and prepositions in which forces seem to play a role, contributing to the growing attention for this central domain. There are of course still many open problems, like the lack of a good understanding of the restriction on force modification in resultative structures that we documented for German, and its interaction with the well-known manner versus result distinction (cf. Levin \& Hovav 2013). How this restriction extends beyond the German data that we presented is still unclear, as well as how our informal explanation can be worked out in a more principled way, substantiating the intricate force-dynamic interactions that we see between word meaning and sentence structure.

\section{References}

Copley, Bridget \& Heidi Harley. 2015. A force-theoretic framework for event structure. Linguistics and Philosophy 38. 103-158. doi:10.1007/s10988-015- 
9168-x.

Croft, William. 2012. Verbs: Aspect and Causal Structure. Oxford: Oxford University Press.

Gärdenfors, Peter. 2000. Conceptual Spaces: The Geometry of Thought. Cambridge, MA: MIT Press.

Gärdenfors, Peter. 2014. The Geometry of Meaning: Semantics Based on Conceptual Spaces. Cambridge, MA: MIT Press.

Jackendoff, Ray. 1990. Semantic Structures. Cambridge, MA: MIT Press.

Kratzer, Angelika. 1996. Severing the external argument from its verb. In Johan Rooryck \& Laurie Zaring (eds.), Phrase Structure and the Lexicon, 109-137. Dordrecht: Kluwer Academic Publishers.

Levin, Beth. 1993. English Verb Classes and Alternations. Chicago: The University of Chicago Press.

Levin, Beth \& Malka Rappaport Hovav. 2013. Lexicalised meaning and manner/result complementarity. In Boban Arsenijevic, Berit Gehrke \& Rafael Marín (eds.), Subatomic Semantics of Event Predicates, 49-70. Dordrecht: Springer.

Parsons, Terence. 1990. Events in the Semantics of English. Cambridge, MA: MIT Press.

Pustejovsky, James. 1991. The syntax of event structure. Cognition 41(1). 47-81. doi:10.1016/0010-0277(91)90032-Y.

Roßdeutscher, Antje \& Tillmann Pross. 2015. Representing force dynamics at the syntax-semantics-interface. Handout at the workshop Formal Semantics Meets Cognitive Semantics, Nijmegen.

Talmy, Leonard. 2000. Toward a Cognitive Semantics: Force Dynamics in Language and Cognition. Cambridge, MA: MIT Press.

Van Lambalgen, Michiel \& Fritz Hamm. 2008. The Proper Treatment of Events. Oxford: Blackwell Publishing.

Wolff, Phillip. 2007. Representing causation. Journal of Experimental Psychology. General 136(1). 82-111. doi:10.1037/0096-3445.136.1.82.

Zwarts, Joost \& Yoad Winter. 2000. Vector space semantics: A model-theoretic analysis of locative prepositions. Journal of Logic, Language and Information 9(2). 171-213. doi:10.1023/A:1008384416604.

Anja Goldschmidt

Utrecht University

Trans 10

NL-3512JK Utrecht

A.Goldschmidt@uu.nl
Joost Zwarts

Utrecht University

Trans 10

NL-3512JK Utrecht

J.Zwarts@uu.nl 